\title{
UCP2 and ANT differently modulate proton-leak in brain mitochondria of long-term hyperglycemic and recurrent hypoglycemic rats
}

\author{
Susana Cardoso • Maria S. Santos • \\ António Moreno • Paula I. Moreira
}

Received: 27 November 2012 / Accepted: 6 February 2013 /Published online: 17 March 2013

(C) Springer Science+Business Media New York 2013

\begin{abstract}
A growing body of evidence suggests that mitochondrial proton-leak functions as a regulator of reactive oxygen species production and its modulation may limit oxidative injury to tissues. The main purpose of this work was to characterize the proton-leak of brain cortical mitochondria from long-term hyperglycemic and insulininduced recurrent hypoglycemic rats through the modulation of the uncoupling protein 2 (UCP2) and adenine nucleotide translocator (ANT). Streptozotocin-induced diabetic rats were treated subcutaneously with twice-daily insulin injections during 2 weeks to induce the hypoglycemic episodes. No differences in the basal proton-leak, UCP2 and ANT protein levels were observed between the experimental groups. Mitochondria from recurrent hypoglycemic rats presented a decrease in proton-leak in the presence of GDP, a specific UCP2 inhibitor, while an increase in proton-leak was observed in the presence of linoleic acid, a proton-leak activator, this effect being reverted by the
\end{abstract}

S. Cardoso $\cdot$ M. S. Santos $\cdot$ A. Moreno $\cdot$ P. I. Moreira

Center for Neuroscience and Cell Biology, University of Coimbra,

3004-517 Coimbra, Portugal

S. Cardoso $\cdot$ M. S. Santos $\cdot$ A. Moreno

Department of Life Sciences - Faculty of Sciences and

Technology, University of Coimbra, 3004-517 Coimbra, Portugal

A. Moreno

Institute of Marine Research, University of Coimbra, 3004-517

Coimbra, Portugal

P. I. Moreira $(\bowtie)$

Laboratory of Physiology, Faculty of Medicine,

University of Coimbra, 3004-548 Coimbra, Portugal

e-mail: venta@ci.uc.pt

P. I. Moreira

e-mail: pimoreira@fmed.uc.pt simultaneous addition of GDP. Mitochondria from longterm hyperglycemic rats showed an enhanced susceptibility to ANT modulation as demonstrated by the complete inhibition of basal and linoleic acid-induced proton-leak caused by the ANT specific inhibitor carboxyatractyloside. Our results show that recurrent-hypoglycemia renders mitochondria more susceptible to UCPs modulation while the protonleak of long-term hyperglycemic rats is mainly modulated by ANT, which suggest that brain cortical mitochondria have distinct adaptation mechanisms in face of different metabolic insults.

Keywords ANT $\cdot$ Cortical brain mitochondria $\cdot$ Long-term hyperglycemia $\cdot$ Proton-leak $\cdot$ Recurrent hypoglycemia . UCP2

\section{Introduction}

Coincident with the use of oxygen for aerobic respiration is the generation of reactive oxygen species (ROS), whose levels are controlled by endogenous cellular antioxidants to keep oxidative homeostasis. This is especially relevant at the mitochondrial level since the respiratory chain is the major site for ROS production (Naudi et al. 2012). Besides the antioxidant defense system, the respiratory chain itself can modulate mitochondrial ROS production through specific proteins (Leloup et al. 2011). Mitochondrial protonleak is a basal or induced permeability of the mitochondrial inner membrane, resulting in partial dissipation of the transmembrane electrochemical gradient $\left(\Delta \Psi_{\mathrm{m}}\right)$ and uncoupling of substrate oxidation from ATP synthesis (Brookes 2005). Since mitochondrial-derived ATP is essential for cellular functions such uncoupling is often regarded as being detrimental. However, a growing body of evidence suggests that 
a low level of proton-leak may have a cytoprotective role (Mattiasson et al. 2003; Teshima et al. 2003; Hoerter et al. 2004), possibly because mild $\Delta \Psi_{\mathrm{m}}$ dissipation diminished ROS generation, and prevented mitochondrial $\mathrm{Ca}^{2+}$ overload (Korshunov et al. 1997; Votyakova and Reynolds 2001; Miwa and Brand 2003). Two groups of mitochondrial carrier proteins contribute significantly to proton leak, uncoupling proteins (UCPs) and the adenine nucleotide translocator (ANT) (Naudi et al. 2012; Brand and Esteves 2005). The initial connection between UCPs and ROS came from a study made by Nègre-Salvayre et al. (Negre-Salvayre et al. 1997) revealing that the inhibition of UCP1 activated the formation of ROS in brown fat mitochondria. Subsequent data suggested that UCP activity may lead to an increase in proton conductance through the interaction with superoxide anion $\left(\mathrm{O}_{2}{ }^{-\bullet}\right)$ (Echtay et al. 2002) or ROS products (Echtay et al. 2003). The suggestion that an increased ROS production would lead to mild-uncoupling that, in turn, would decrease ROS formation, placed neuronal UCPs as possible important brain damage modifiers in protecting against oxidative stress (Cardoso et al. 2011). Likewise, mitochondrial proton-leak mediated by ANT has been reported to occur in the presence of lipid peroxidation products leading to a feedback mechanism to protect against ROS damage (Echtay et al. 2003).

Chronic hyperglycemia is the hallmark of diabetes and associated complications. High glucose-induced excessive ROS production has been considered to play an important role in the onset and progression of diabetes mellitus (DM) (Rains and Jain 2011). DM is a complex metabolic disorder characterized by defects in the body's ability to control glucose and insulin homeostasis (Rains and Jain 2011). Accumulating evidence shows that DM is often associated with complications in the central nervous system which can lead to cognitive deficits and dementia (Gispen and Biessels 2000). The increased risk for dementia in diabetic patients may result from direct effects of hyperglycemia, hypoglycemia and disrupted insulin signaling in the brain or indirect ischemic effects of diabetes-promoted cerebrovascular disease (Craft 2009; Wrighten et al. 2009). Despite the presence of three UCPs isoforms in the brain, UCP2 has been the major focus of studies concerning brain damage and neurodegeneration. It was previously demonstrated that an increase in brain UCP2 expression was correlated with the survival of cortical neurons in conditions of oxygen and glucose deprivation, a situation that was associated with a decrease in mitochondrial ROS production (Mattiasson et al. 2003). Despite this evidence, the role of UCP2 in diabetesinduced brain damage remains elusive.

In the present study, our main goal was to characterize the proton-leak of brain cortical mitochondria, through UCP2 and ANT modulation, exposed to insulin-induced recurrent hypoglycemia and/or long-term hyperglycemia, conditions that occur in type 1 diabetic patients under insulin therapy.

\section{Materials and methods}

Chemicals Streptozotocin was obtained from Sigma (Portugal). Insulin (Humulin NPH) was obtained from Eli Lilly and Company (USA). Anti-UCP2 antibody was obtained from Calbiochem-EMD Chemicals (Gibbstown, USA). All the other chemicals were of the highest grade of purity commercially available.

Animals treatment Male Wistar rats (2-month-old) were housed in our animal colony (Laboratory Research Center, Faculty of Medicine, University of Coimbra) and were maintained under controlled light (12 hday/night cycle) and humidity with free access to water and powdered rodent chow (except in the fasting period). Rats were deprived of food overnight and were randomly divided in two groups. One group received an i.p. injection of STZ $(50 \mathrm{mg} / \mathrm{kg}$ body weight) freshly dissolved in citrate $100 \mathrm{mM}, \mathrm{pH} 4.5$. The volume administered was always $0.5 \mathrm{ml} / 200 \mathrm{~g}$ animal body weight. The control group received an i.p. injection with an equal volume of citrate (vehicle). In the following $24 \mathrm{~h}$, animals were orally fed with glycosylated serum in order to avoid hypoglycemia resulting from the massive destruction of $\beta$-cells and release of intracellular insulin associated with STZ treatment (Moreira et al. 2005). Three days after STZ administration, the tail vein blood glucose levels were measured in all animals and those presenting levels above $250 \mathrm{mg} / \mathrm{dl}$ were considered diabetic. After 3 months of the induction of diabetes, the STZ-diabetic rats were randomly divided in two groups and one group was subjected to recurrent hypoglycemia achieved by twice-daily injections of insulin [s.c., dose adjusted to blood glucose levels] during 2 weeks. Animals handling and sacrifice followed the procedures approved by the Federation of European Laboratory Animal Science Associations (FELASA).

Determination of blood glucose and glycated hemoglobin levels Blood glucose concentration was determined from the tail vein using a commercial glucometer (Glucometer-Elite, Bayer, Portugal). Hemoglobin A1C (HbA1c) levels were determined using Systems SYNCHRON CX 4 (Beckman). This system utilizes two cartridges, $\mathrm{Hb}$ and $\mathrm{A} 1 \mathrm{c}$ to determine Alc concentration as a percentage of total $\mathrm{Hb}$. The hemoglobin is measured by a colorimetric method and the A1c concentration by a turbidimetric immunoinhibition method.

Preparation of mitochondrial fraction Brain cortical mitochondria were isolated from rats according to (Moreira et al. 2001, 2002). In brief, the rat was decapitated, and the cortex was rapidly removed, washed, minced, and homogenized at $4{ }^{\circ} \mathrm{C}$ in $10 \mathrm{ml}$ of isolation medium $(225 \mathrm{mM}$ mannitol, $75 \mathrm{mM}$ sucrose, $5 \mathrm{mM}$ HEPES, $1 \mathrm{mM}$ EGTA, $1 \mathrm{mg} / \mathrm{ml}$ BSA, pH7.4) containing $5 \mathrm{mg}$ of bacterial protease type 
VIII (Subtilisin). Single brain homogenates were brought to $20 \mathrm{ml}$ and then centrifuged at $2500 \mathrm{rpm}$ (Sorvall RC-5B Refrigerated Superspeed Centrifuge) for $5 \mathrm{~min}$. The pellet, including the fluffy synaptosomal layer, was resuspended in $10 \mathrm{ml}$ of the isolation medium containing $0.02 \%$ digitonin and centrifuged at $10000 \mathrm{rpm}$ for $10 \mathrm{~min}$. The brown mitochondrial pellet without the synaptosomal layer was then resuspended again in $10 \mathrm{ml}$ of medium and centrifuged at $10000 \mathrm{rpm}$ for $5 \mathrm{~min}$. The pellet was resuspended in $10 \mathrm{ml}$ of washing medium (225 mM mannitol, $75 \mathrm{mM}$ sucrose, $5 \mathrm{mM}$ HEPES, pH7.4) and centrifuged at $10000 \mathrm{rpm}$ for $5 \mathrm{~min}$. The final mitochondrial pellet was resuspended in the washing medium and mitochondrial protein was determined by the biuret method calibrated with BSA (Gornall et al. 1949).

Proton-leak measurements In the presence of oligomycin (ATP synthase inhibitor) the respiration rate is proportional to the rate of protons leaking through the mitochondrial inner membrane. The kinetic response of the proton-conductance pathway to its driving force can therefore be measured as the relationship between respiration rate and mitochondrial membrane potential, when the potential changes by titration with electron transport chain inhibitors. Briefly, oxygen consumption and inner membrane potential were simultaneously recorded using a Clark-type oxygen electrode and a $\mathrm{TPP}^{+}$ electrode, respectively. Cortical brain mitochondria $(0.8 \mathrm{mg})$ were incubated in $1 \mathrm{ml}$ of reaction medium with $2 \mu \mathrm{M}$ of oligomycin, $3 \mu \mathrm{M}$ of rotenone and $80 \mathrm{ng} / \mathrm{ml}$ of nigericin (added to abolish $\Delta \mathrm{pH}$ so that the total proton-motive force can be equal to $\Delta \Psi_{\mathrm{m}}$ ). Experiments started with succinate, a substrate for complex II. Oxygen consumption and electric potential were progressively inhibited with sequential additions of malonate (inhibitor of complex II) up to a total of $2 \mathrm{mM}$. At the end of each experiment, $0.2 \mu \mathrm{M}$ of valinomycin was added to dissipate the $\Delta \Psi_{\mathrm{m}}$ (Cadenas et al. 2000). Titration curves for stimulation and inhibition of proton-leak were also assessed. To study UCP2 activation, $5 \mu \mathrm{M}$ linoleic acid (a known UCP2 activator) (Jaburek et al. 2004) was added before the additions of malonate. In the experiments involving proton-leak inhibition, the UCP2 inhibitor guanosine diphosphate (GDP; $750 \mu \mathrm{M})$ (Echtay et al. 2003) or the potent and specific ANT inhibitor carboxyatractyloside (CAtr; $1 \mu \mathrm{M}$ ) were added to the reaction medium.

Western blotting Mitochondrial fractions were homogenized in RIPA buffer (50 mM Tris- $\mathrm{HCl}, 150 \mathrm{mM} \mathrm{NaCl}, 1 \mathrm{mM}$ EDTA, $1 \%$ Triton-x100, $1 \%$ deoxycorticosterone, $0.1 \%$ SDS), protease and phosphatase inhibitors (commercial protease and phosphatase inhibitors cocktails from Roche), 0 . $1 \mathrm{~mol} / \mathrm{L}$ phenylmethylsulfonyl fluoride, $0.2 \mathrm{~mol} / \mathrm{L}$ dithiothreitol, and samples ( $80 \mu \mathrm{g}$ of protein per lane) were run on $10 \%$ SDS-polyacrylamide gels. After electrophoresis, proteins were transferred to PVDF membranes, and blocked membranes ( $1 \mathrm{~h}$ in $5 \% \mathrm{BSA}$ and $0.1 \%$ Tween in TBS for $1 \mathrm{~h}$ at room temperature) were incubated overnight at $4{ }^{\circ} \mathrm{C}$ with a rabbit anti-UCP2 antibody (1:1000, Calbiochem), a goat antiANT2/3 (1:100, Santa Cruz) and a rabbit antibody against cytochrome oxidase subunit IV (COX IV- 1:1000, Cell Signaling). The proteins were detected separately with the secondary antibodies, anti-rabbit (1:10000) for UCP2 and COX-IV detection and anti-goat (1:2500) for ANT2/3. COX was used as a loading control. The ECF detection system (GE Healthcare) and Versa Doc imaging system (Bio-Rad) were used and densities from each band were obtained with Quantity One Software (Bio-Rad).

Statistical analysis Data were analyzed and results are presented as mean \pm SEM of the indicated number of experiments. Statistical significance between groups was defined using the non-parametric Mann-Whitney $U$-test. A $p$-value $<0.05$ was considered significant.

\section{Results}

Characterization of the experimental animals

As expected, long-term hyperglycemic rats presented significantly higher levels of glycemia and glycated hemoglobin $\left(\mathrm{Hb}_{\mathrm{AlC}}\right)$ and a significant decrease in body weight when compared with control rats (Table 1). Concerning insulininduced recurrent hypoglycemic animals the values presented in Table 1 reflect the blood glucose levels in the peak of the hypoglycemic episodes. $\mathrm{Hb}_{\mathrm{AlC}}$ levels in the recurrent hypoglycemic rats are similar to those of longterm hyperglycemic rats (Table 1).

The basal proton-leak is similar in mitochondria from the three groups of experimental animals

An energetically significant leak of protons occurs across the mitochondrial inner membranes of eukaryotic cells

Table 1 Characterization of the experimental animal models

\begin{tabular}{lccc}
\hline & Control & STZ & Hypoglycemia \\
\hline Body weight $(\mathrm{g})$ & $444 \pm 5.3$ & $300 \pm 8.1^{* * *}$ & $326 \pm 7.8^{* * *}$ \\
Brain weight $(\mathrm{g})$ & $2.06 \pm 0.03$ & $2.13 \pm 0.07$ & $2.21 \pm 0.12$ \\
Glucose $(\mathrm{mg} / \mathrm{dl})$ & $123 \pm 5.1$ & $467 \pm 18.8^{* * *}$ & $48 \pm 5.6^{* * * ;+++}$ \\
$\mathrm{Hb}_{\mathrm{A} 1 \mathrm{C}}(\%)$ & $3.6 \pm 0.03$ & $8.6 \pm 0.48^{* *}$ & $7.4 \pm 0.13^{* *}$ \\
\hline
\end{tabular}

Data are the means \pm SEM of 6-8 animals from each condition studied. Statistical significance: ${ }^{* * *} p<0.001 ;{ }^{* *} p<0.01$ when compared with control rats; ${ }^{+++} p<0.001$ when compared with STZ-treated rats. $\mathrm{Hb}_{\mathrm{A} 1 \mathrm{C}^{-}}$glycated hemoglobin 
(Stuart et al. 1999). Figure 1a shows that the proton conductance of mitochondria from the three groups of experimental animals is similar, although a modest increase in oxygen consumption in the long-term hyperglycemic mitochondrial preparation is observed.

Mitochondria from long-term hyperglycemic and recurrent-hypoglycemic animals do not present significant alterations in UCP2 and ANT protein levels

The uncoupling or proton-leak can be mediated by UCPs and by other mitochondrial inner membrane proteins such as the ANT, which is involved in the modulation of mitochondrial energy efficiency and responsible for half to two-thirds of basal proton conductance (Brand et al. 2005). In our work, no statistically significant alterations were observed in UCP2 protein levels (Fig. 1b), and in the ANT protein levels (Fig. 1c) of both brain cortical mitochondria from longterm hyperglycemic and recurrent-hypoglycemic rats.

Mitochondria from long-term hyperglycemic and recurrent-hypoglycemic rats present a distinct proton-leak profile

The effect of fatty acids-mediated uncoupling through UCP2 and ANT was evaluated by using linoleic acid. A significant linoleic acid-induced stimulation of proton-leak was observed in cortical mitochondria from recurrent hypoglycemic rats, as demonstrated by the shift of the titration curve to the left (Fig. 2c). This effect was confirmed by the higher oxygen consumption needed to achieve the same value of $\Delta \Psi_{\mathrm{m}}$, when compared with the basal situation (Fig. 2c). In the control a

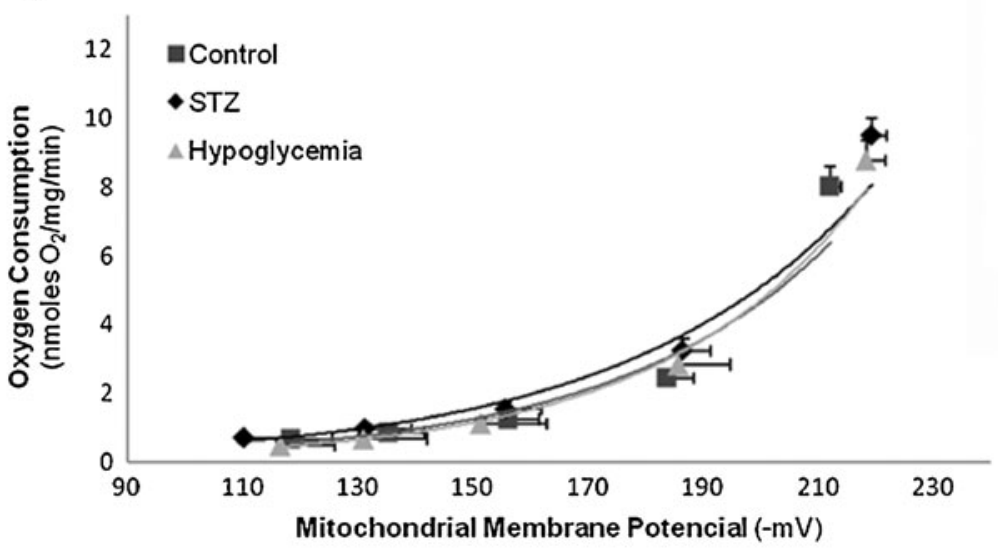

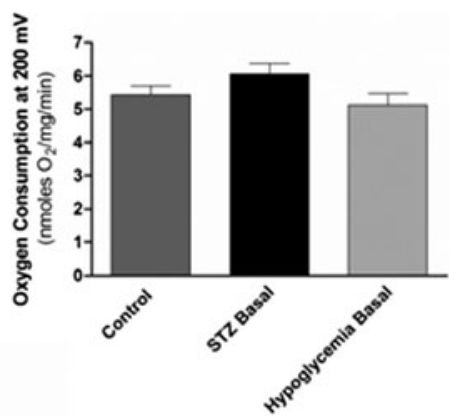

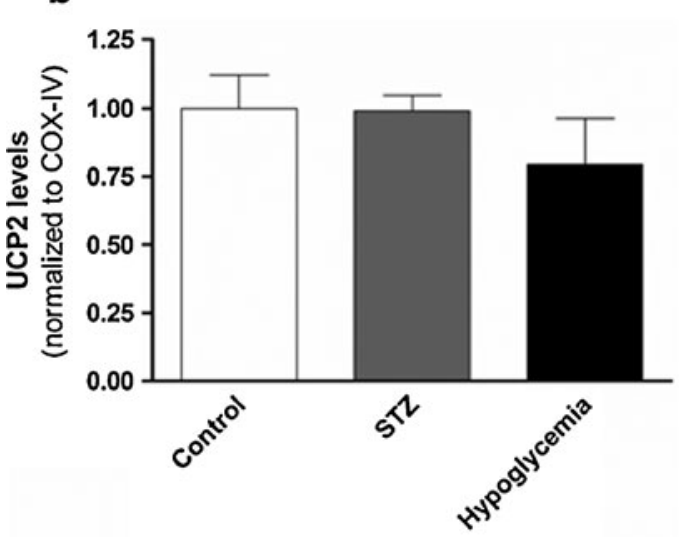

Anti UCP2 33 kDa

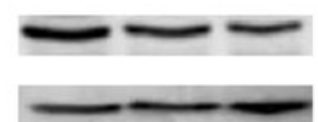

Anti COX-IV $\sim 17 \mathrm{kDa}$

Fig. 1 Effect of STZ-induced long-term hyperglycemia and insulin-induced recurrent hypoglycemia in basal proton-leak (a) and UCP2 (b) and ANT (c) protein levels of brain cortical mitochondria. (a) Titration curves to compare basal proton-leak between the experimental conditions. Bar graphs on the right show the mitochondrial proton-leak at $-200 \mathrm{mV}$. The
C

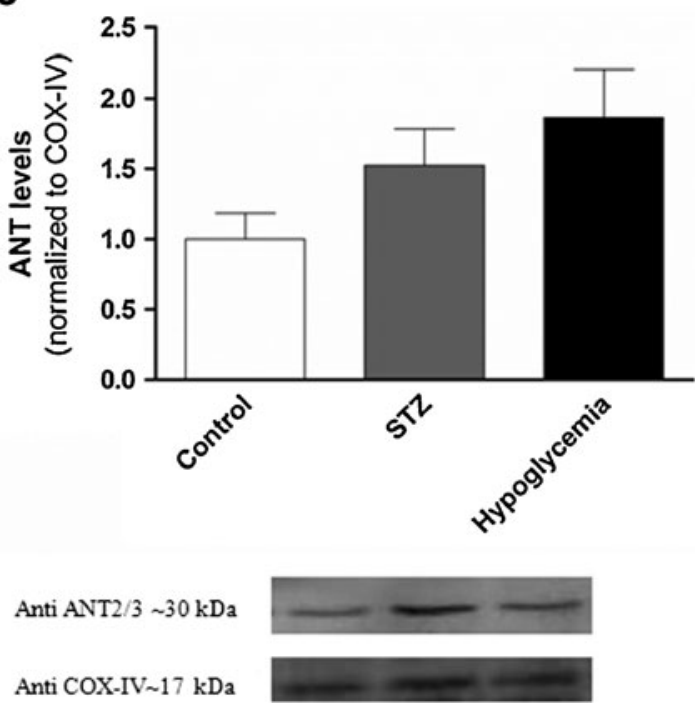

value of $-200 \mathrm{mV}$ was chosen to perform these comparisons since it is the value that better represents the median tendency of all curves. Data are the mean \pm SEM of 6-8 animals from each condition studied. Mitochondrial (b) UCP2 and (c) ANT protein levels. Data are the mean \pm SEM of 4-5 animals from each condition studied 
(Fig. 2a) and long-term hyperglycemic (Fig. 2b) mitochondrial preparations a slight increase in linoleic acid-induced proton-leak stimulation was observed (both titration curves present a modest increase in oxygen consumption for the same value of $\Delta \Psi_{\mathrm{m}}$ ).

Additionally, to determine the relative contribution of either UCPs or ANT to proton-leak, experiments with GDP or CAtr, inhibitors of UCPs and ANT, respectively, were performed. Figures $3 a$ and $b$ show that GDP did no affect proton-leak of control and long-term hyperglycemic mitochondria. However, CAtr induced an inhibitory effect in proton-leak of control and long-term hyperglycemic mitochondria as shown by the shift of the titration curve to the right, which was accompanied by a lower oxygen consumption for the same $\Delta \Psi_{\mathrm{m}}$, suggesting that the proton-leak of mitochondria from these two experimental groups are modulated by ANT. Mitochondria from the recurrent hypoglycemic animals a

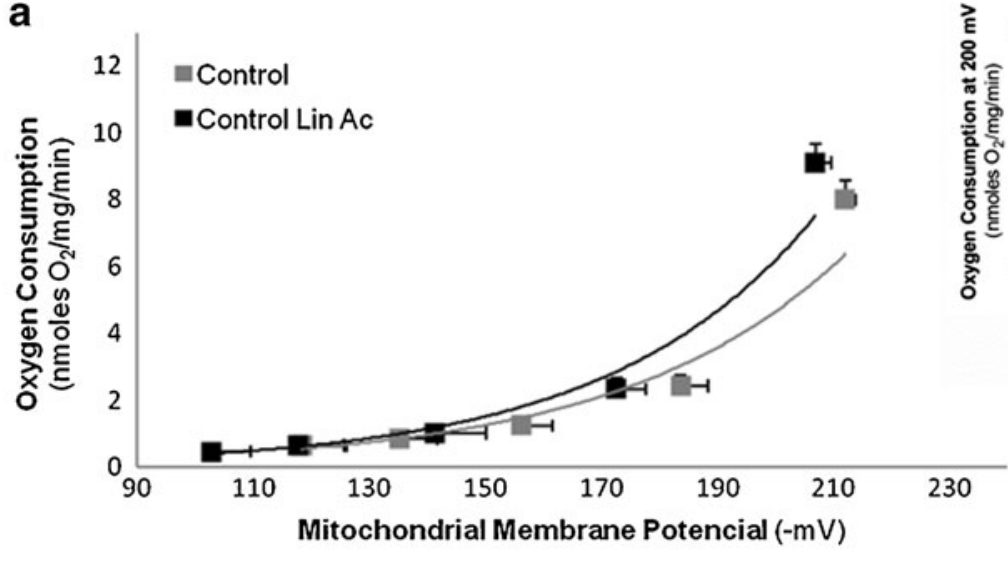

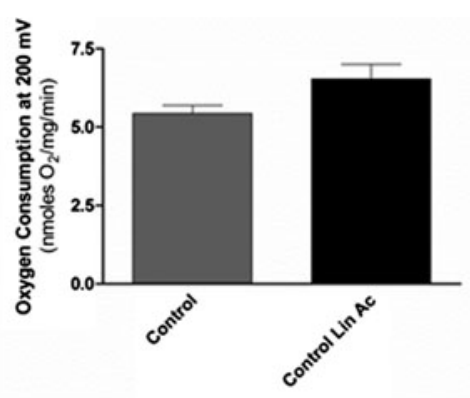

b
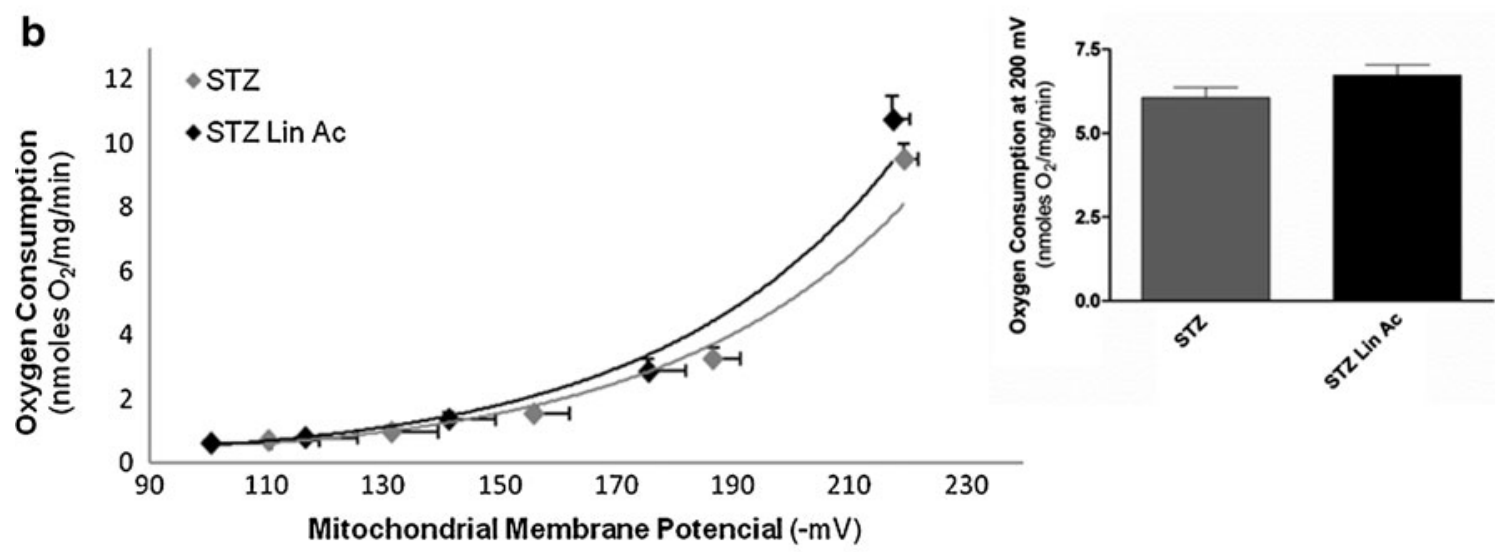

C
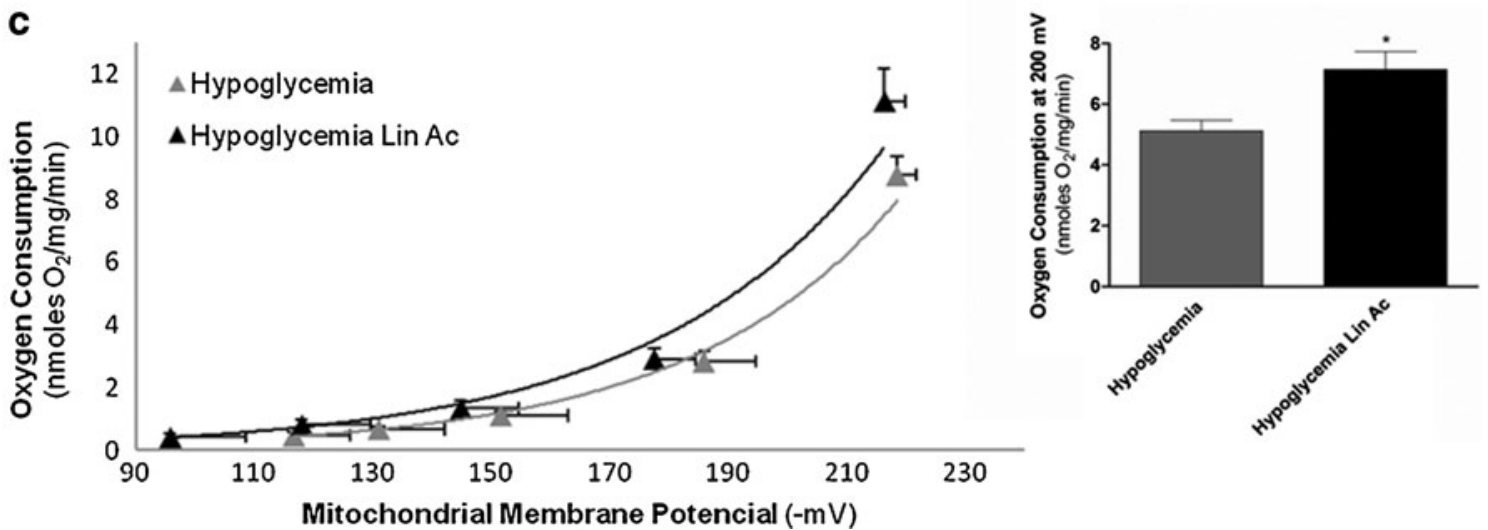

Fig. 2 Effect of linoleic acid on the rate of proton-leak in brain cortical mitochondria from control (a), STZ-induced long-term hyperglycemic (b) and insulin-induced recurrent hypoglycemic (c) animals. Bar graphs on the right show the mitochondrial proton-leak at $-200 \mathrm{mV}$. The value of $-200 \mathrm{mV}$ was chosen to perform these comparisons since it is the value that better represents the median tendency of all curves. Data are the mean \pm SEM of 6 -8 animals from each condition studied. ${ }^{*} p<0.05$ when compared with hypoglycemic brain cortical mitochondria 
show a high susceptibility to GDP- and CAtr-mediated proton-leak inhibition that was characterized by a significant shift of the titration curve to the right and a low rate of oxygen consumption (Fig. 3c) suggesting that both UCPs and ANT modulate proton-leak in hypoglycemic animals. This observation was further confirmed by evaluating the ability of both inhibitors to neutralize the linoleic acid-induced increase in proton-leak. In fact, the co-addition of GDP and linoleic acid reduced the linoleic acid-mediated stimulation of UCPs activity in mitochondria from insulin-induced recurrent hypoglycemia (Fig. 4c) but not in control (Fig. 4a) and long-term hyperglycemic (Fig. 4b) rats. Moreover, co-incubation of CAtr and linoleic acid abolished the stimulatory effect of linoleic acid in control and long-term hyperglycemic rats (Figs. 5a and b).
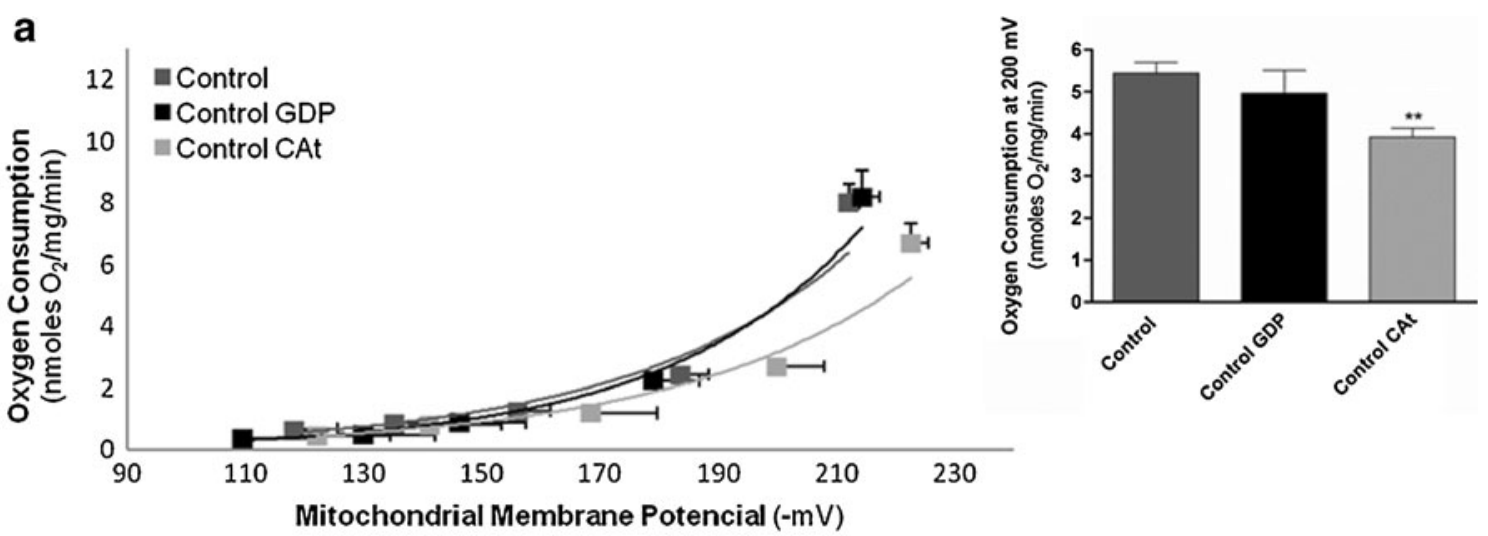

b
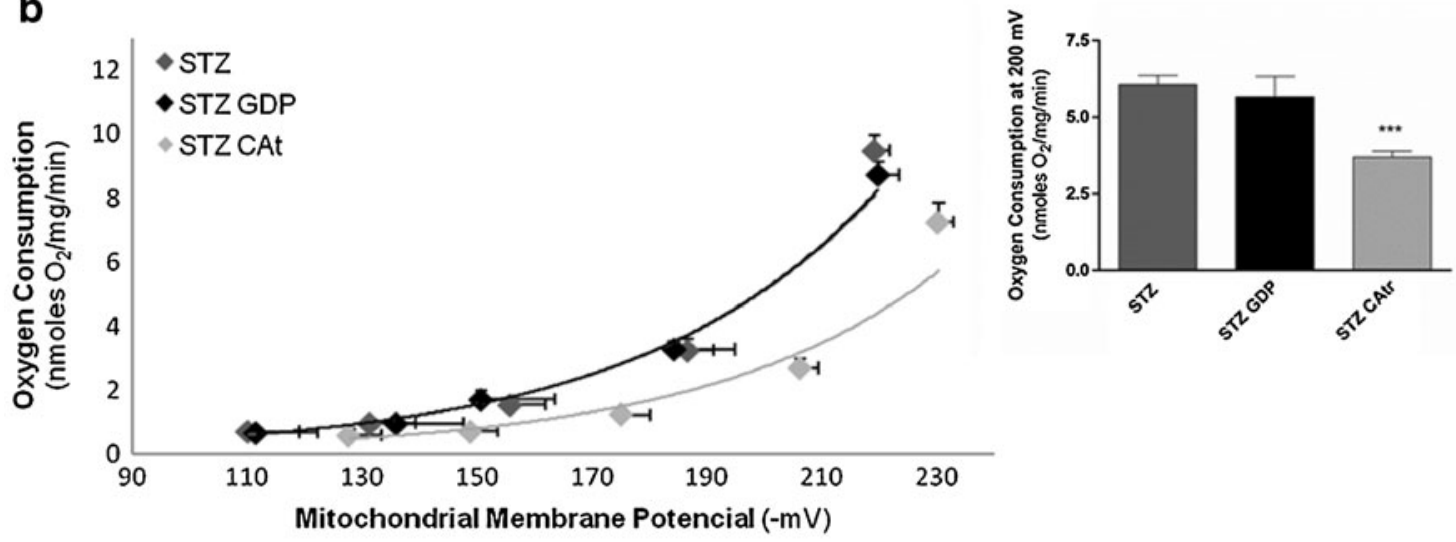

C
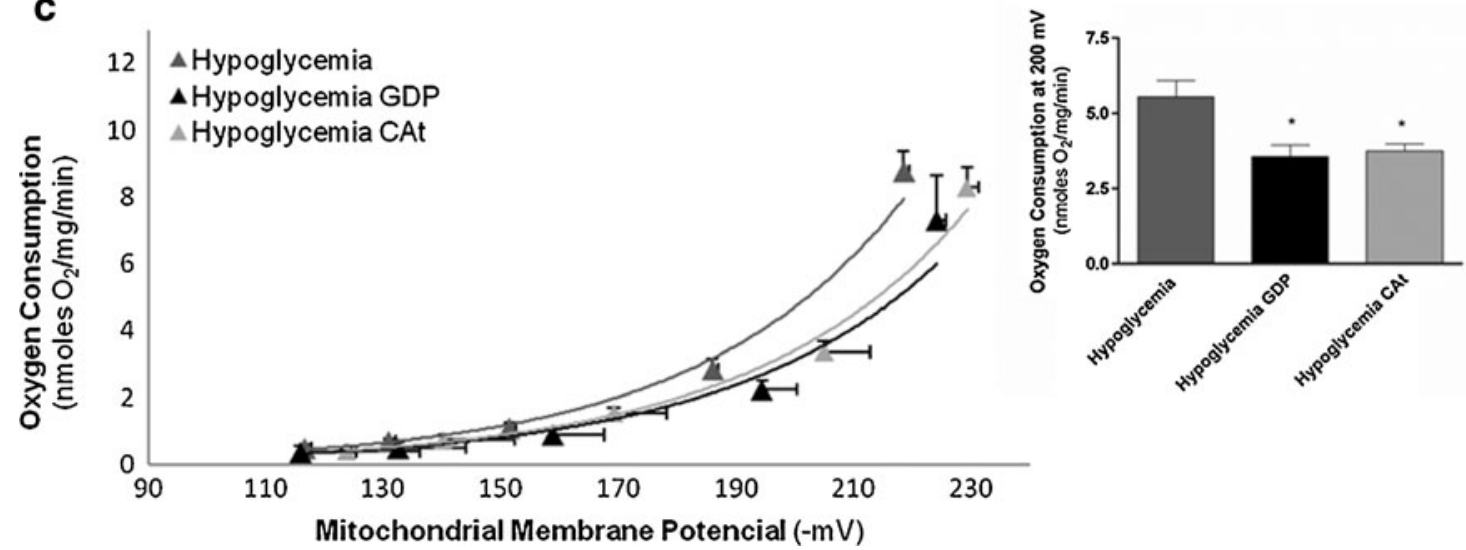

Fig. 3 Effect of GDP and carboxyatractryloside on the rate of proton-leak in brain cortical mitochondria from control (a), STZ-induced long-term hyperglycemic (b) and insulin-induced recurrent hypoglycemic (c) animals. Bar graphs on the right show the mitochondrial proton-leak at $-200 \mathrm{mV}$. The value of $-200 \mathrm{mV}$ was chosen to perform these comparisons since it is the value that better represents the median tendency of all curves. Data are the mean \pm SEM of $6-8$ animals from each condition studied. ${ }^{* * *} \mathrm{p}<0.001$ when compared with STZ brain cortical mitochondria; ${ }^{* *} \mathrm{p}<0.01$ when compared with control brain cortical mitochondria; ${ }^{*} p<0.05$ when compared with hypoglycemic brain cortical mitochondria 


\section{Discussion}

To mimic blood glucose levels fluctuations that often occur in type 1 diabetic patients under intensive insulin therapy (Jacob et al. 1999; Herzog et al. 2008), we induced recurrent hypoglycemic episodes in long-term hyperglycemic rats. To characterize brain mitochondrial uncoupling under recurrent hypoglycemia and/or long-term hyperglycemia, we investigated two mitochondrial carrier proteins, UCP2 and ANT, which modulate mitochondrial proton-leak. Mitochondria from recurrent hypoglycemic rats exhibited a higher linoleic acid-induced proton-leak that was inhibited by GDP (Fig. 4c), whereas mitochondria from long-term hyperglycemic rats were more susceptible to ANT modulation (Fig. 3b). Mitochondria are main energy producers and it is believed that an adaption of mitochondrial efficiency allows the organisms to respond to a

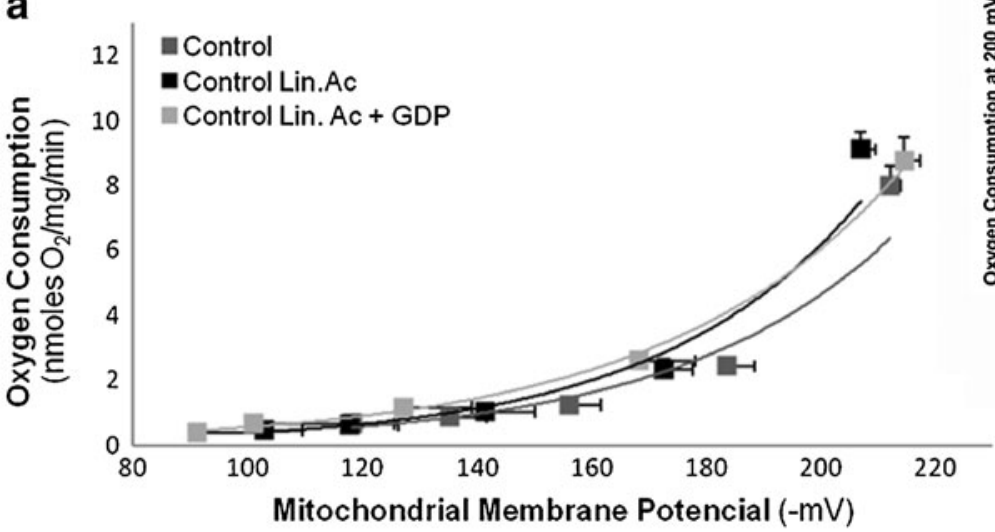

b

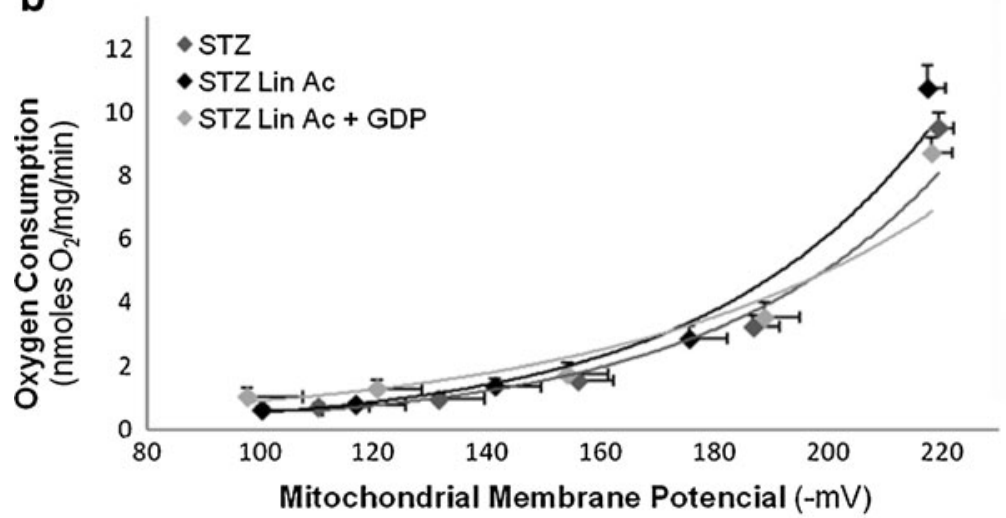

C

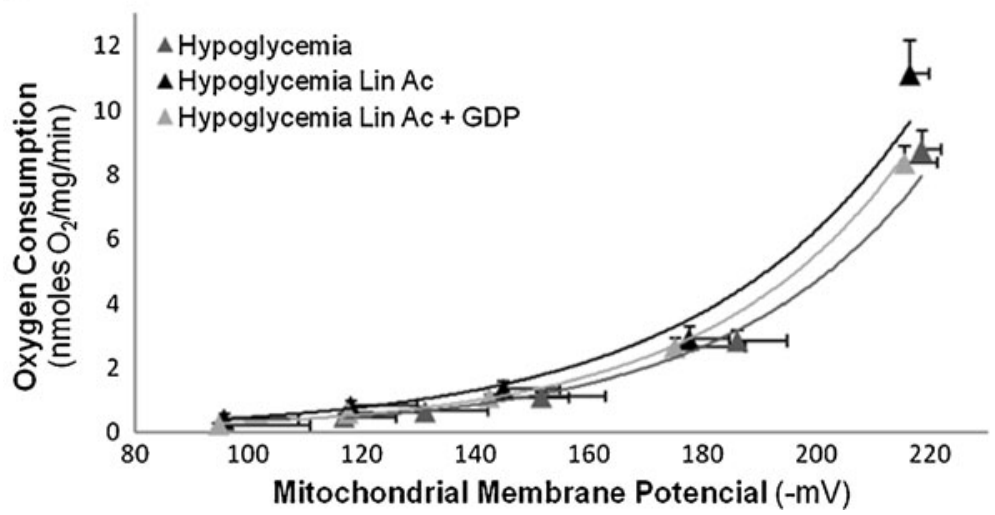

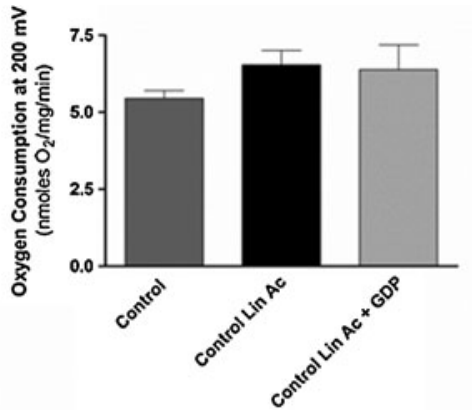
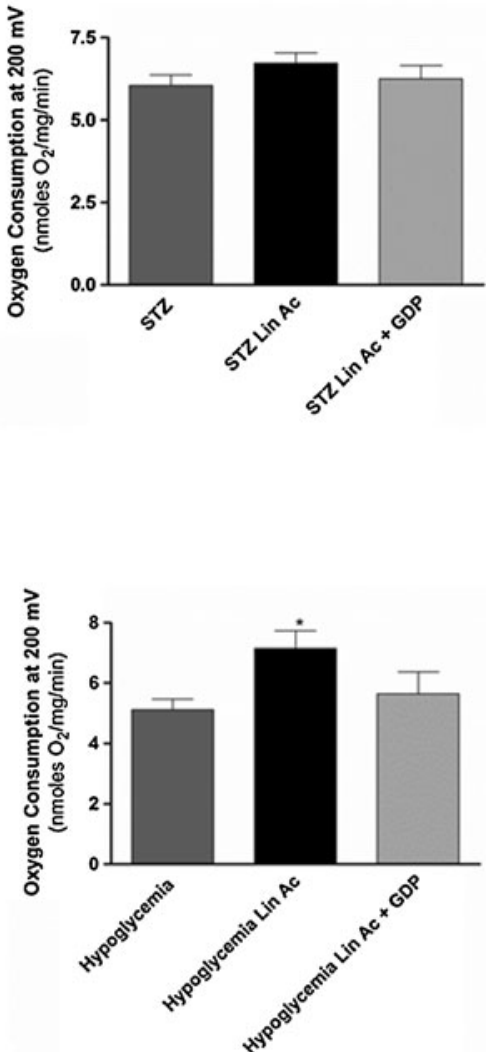

Fig. 4 Effect of GDP on the rate of linoleic acid-induced proton-leak in brain cortical mitochondria from control (a), STZ-induced long-term hyperglycemic (b) and insulin-induced recurrent hypoglycemic (c) animals. Bar graphs on the right show the mitochondrial proton-leak at $-200 \mathrm{mV}$. The value of $-200 \mathrm{mV}$ was chosen to perform these comparisons since it is the value that better represents the median tendency of all curves. Data are the mean \pm SEM of 6-8 animals from each condition studied. ${ }^{*} \mathrm{p}<0.05$ when compared with hypoglycemic brain cortical mitochondria 
physiological challenges (Trzcionka et al. 2008). Following this line of thought we can speculate that brain cortical mitochondria can also develop adaptation mechanisms in face to distinct metabolic insults.

When ATP synthase (complex V) is not working, either in the absence of ADP or in the presence of oligomycin (which blocks the passage of protons through the $\mathrm{F}_{\mathrm{O}}$ portion of the ATPase), all protons pumped out of the matrix move back into the mitochondria through other proton conductance routes. Flux through this nonproductive proton conductance pathway has been termed "proton-leak" (Stuart et al. 1999). Therefore, proton-leak is greatest under nonphosphorylating conditions. When ADP and $\mathrm{P}_{\mathrm{i}}$ are present (state 3 of respiration), ATP synthase is the favored route of proton reentry to the matrix, and thus proton-leak is essentially switched off when energy is needed. An increase in state 4 of respiration alone or a decrease in $\Delta \Psi_{\mathrm{m}}$ alone is often used as a surrogate for protonleak changes (Brookes 2005). Previous data from our laboratory shows that mitochondria from rats exposed to metabolic insults as those described in this study, presented no significant differences in the respiratory chain and phosphorylation system (Cardoso et al. 2012), which suggest that basal protonleak is similar in all groups of experimental animals. Nonetheless, a titration of the respiratory chain activity in the presence of oligomycin, with simultaneous measurement of respiration rate and $\Delta \Psi_{\mathrm{m}}$, remains the only effective measure of proton-leak (Brookes 2005). Accordingly, our titration experiments showed no statistically significant changes in proton-leak in mitochondria from the three experimental groups (Figs. 1a). Furthermore, no significant alterations in UCP2 protein levels were observed in our experimental groups (Fig. 1b). Although proton-leak is associated with UCPs mRNA and protein levels this relation is not straightforward. It was previously shown that overnight starvation increased UCP $2 / 3$ mRNA and protein levels, but did not alter mitochondrial proton-leak (Cadenas et al. 1999). (Herlein et al. 2009) demonstrated that heart and muscle mitochondria from diabetic animals were actually more coupled compared to control animals despite an increase in UCP3 content, thus demonstrating that expression of UCPs, respiration and proton-leak are not entirely interrelated. There is also the assumption that UCP2 and UCP3 do not increase proton conductance in the absence of activators, as suggested by studies showing that the knockdown of UCP2 or UCP3 did not affect basal proton conductance of mouse mitochondria (Cadenas et al. 2002; Aguirre and Cadenas 2010).

The proton conductance mediated by UCP 2 and UCP 3 is activated by fatty-acids and inhibited by purine nucleotides (Echtay et al. 2001; Chan et al. 2010) and, usually, UCPs remain inactive in the absence of the activators due to their inhibition by physiological concentrations of purine nucleotides (Brand and Esteves 2005; Jaburek et al. 2004). A significant linoleic acid-induced proton-leak stimulation was observed in the recurrent hypoglycemic mitochondria (Fig. 2c), which was completely inhibited by the coincubation with GDP (Fig. 4c). In agreement, GDP strongly inhibited proton-conductance in mitochondria from the recurrent hypoglycemic rats (Fig. 3c), a situation that was not observed in mitochondria from control (Fig. 3a) and longterm hyperglycemic (Fig. 3b) rats. The sensitivity to GDPmediated inhibition of proton-leak appears to be indicative of UCPs involvement in mitochondrial proton conductance (Chan et al. 2010). A previous study demonstrated that heart mitochondria from type 2 diabetic mice were GDP sensitive; however this was not correlated with changes in the expression levels of UCP3 (Boudina et al. 2007). Similarly, we observed that recurrent hypoglycemic mitochondria were susceptible to GDP, although no statistically significant changes were observed in UCP2 protein levels (Fig. 1b).

The proton-leak mediated by ANT is inhibited by CAtr, bongkrekic acid, ADP and ATP (Cadenas et al. 2000; Aguirre and Cadenas 2010). Although no statistically significant changes occurred in ANT protein levels (Fig. 1c), brain cortical mitochondria from all experimental groups were susceptible to CAtr-mediated inhibition of ANT (Figs. 3a, b and c). The strongest effect of CAtr occurred in long-term hyperglycemic mitochondria, as shown by the shift of the titration curve to the right and the significant decrease in oxygen consumption (Fig. 3b). In agreement, CAtr completely prevented the linoleic acid-induced uncoupling in mitochondrial preparations from long-term hyperglycemic rats (Fig. 5b), which may suggest that ANT-induced proton-leak may play a role under hyperglycemic conditions.

The literature shows that uncontrolled blood glucose levels impact brain structure and function potentiating cognitive impairment, with mitochondrial energy metabolism dysfunction and oxidative stress being recognized as main players in diabetes and related complications (McGowan et al. 2006; Moreira et al. 2009; Cardoso et al. 2010). The suggestion that UCPs have a role in diabetes came from studies showing that muscle mitochondria from type 2 diabetes patients presented higher state 4 of respiration and proton-leak that were reverted under a tight glycemic control (Rabol et al. 2009). In endothelial cells, a hyperglycemic insult led to a high increase in the production of ROS that did not occur when the $\Delta \Psi_{\mathrm{m}}$ was collapsed by overexpressing the enzyme superoxide dismutase (MnSOD) or UCP1 (Nishikawa et al. 2000), suggesting that UCP1 have an active role in protecting against hyperglycemiainduced mitochondrial ROS production. It was also shown that UCP3 overexpression in dorsal root ganglions (DRG) was able to prevent the sequence of events associated to glucoseinduced neuronal degeneration (Vincent et al. 2004). In addition, the hyperglycemic insult led to the loss of UCP3 expression in DRG neurons suggesting that preventing the loss of UCP3 may avert glucose-induced neuronal injury (Vincent et al. 2004). Further, it was also demonstrated that UCP2 was not 
a

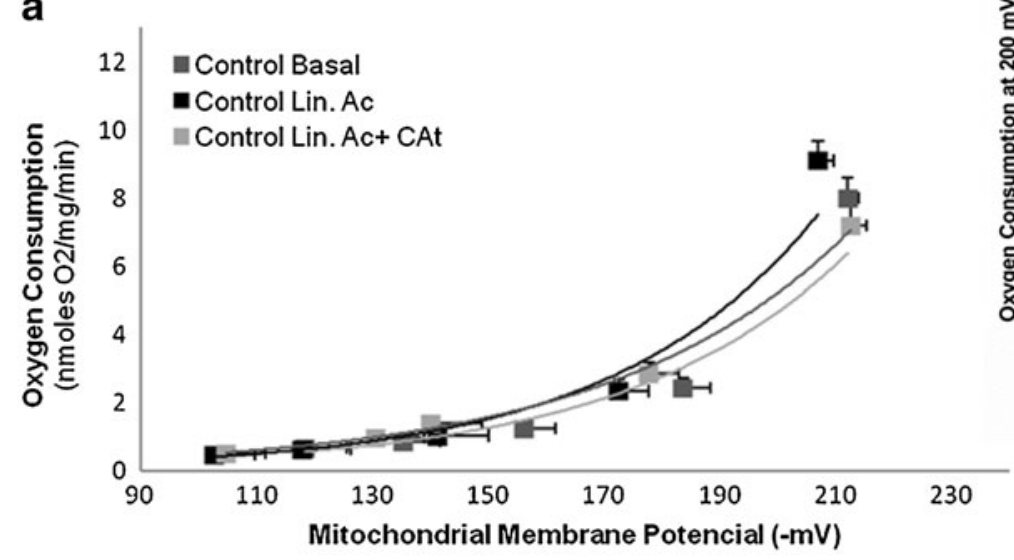

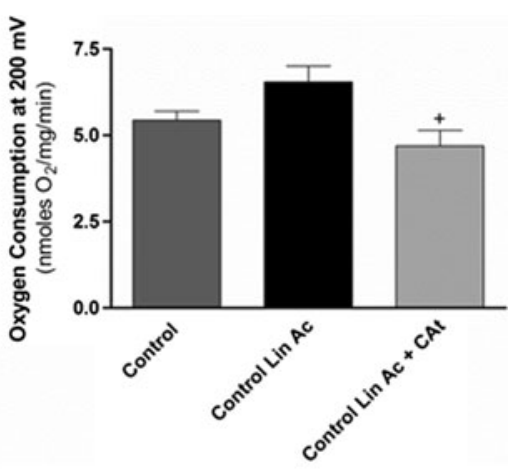

b
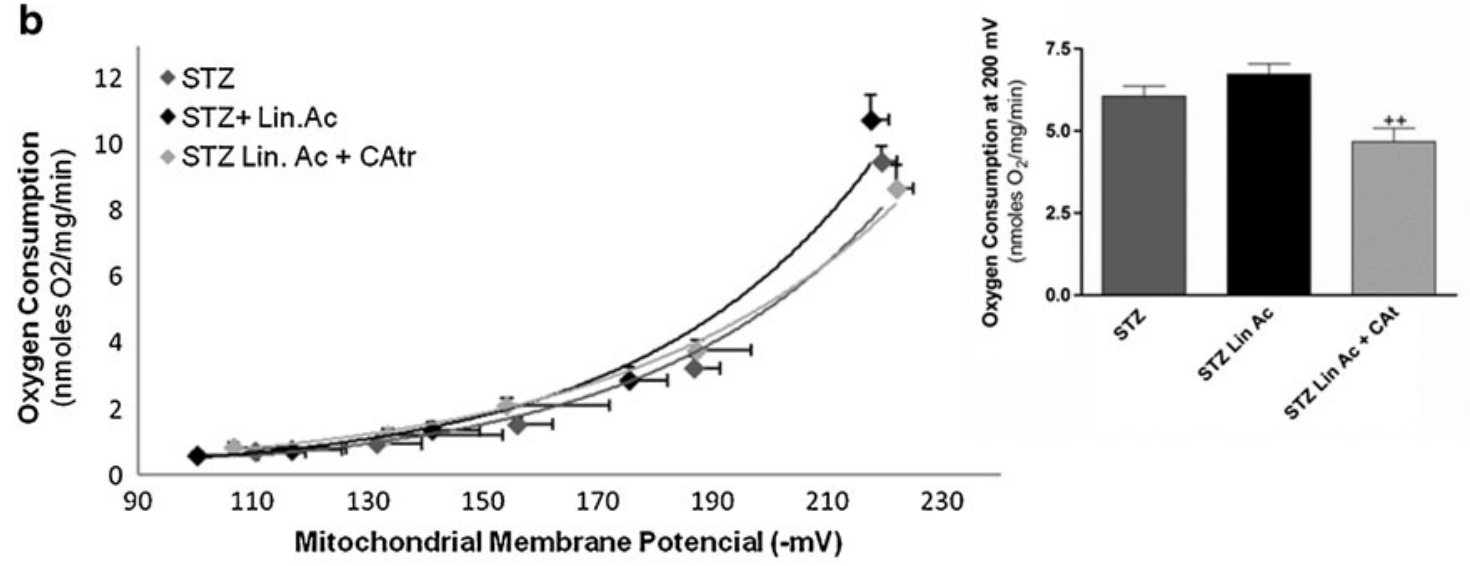

C
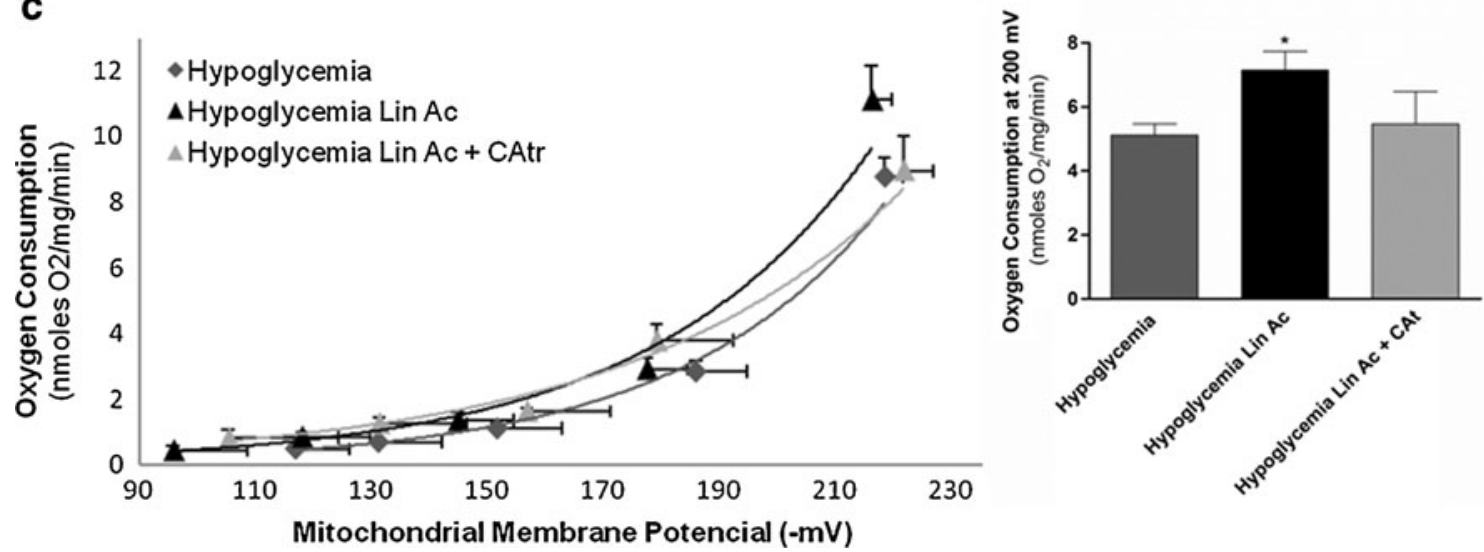

Fig. 5 Effect of carboxyatractryloside on the rate of linoleic acidinduced proton-leak in brain cortical mitochondria from control (a), STZ-induced long-term hyperglycemic (b) and insulin-induced recurrent hypoglycemic (c) animals. Bar graphs on the right show the mitochondrial proton-leak at $-200 \mathrm{mV}$. The value of $-200 \mathrm{mV}$ was chosen to perform these comparisons since it is the value that better represents the

activated directly by $\mathrm{O}_{2}^{-\bullet}$ but rather, the peroxidation of lipids initiated by $\mathrm{O}_{2}^{-\bullet}$ led to an increase in UCP2-mediated proton translocation (Murphy et al. 2003). This lipid peroxidemediated activation of UCP2 is believed to promote egress of fatty acid peroxides from the matrix to the intermembrane space of mitochondria thus making mitochondrial DNA, median tendency of all curves. Data are the mean \pm SEM of 6-8 animals from each condition studied. ${ }^{+} p<0.05$ when compared with control brain cortical mitochondria plus linoleic acid; ${ }^{++} \mathrm{p}<0.01$ when compared with STZ brain cortical mitochondria plus linoleic acid; ${ }^{*} p<0.05$ when compared with hypoglycemic brain cortical mitochondria

aconitase and other mitochondrial matrix-localized components less vulnerable to oxidative damage (Jaburek et al. 2004; Goglia and Skulachev 2003). More recently, evidence suggested that the presence of both free fatty acids and $\mathrm{O}_{2}{ }^{-}$ seem to be required for UCP-mediated uncoupling, whereas $\mathrm{O}_{2}^{-\bullet}$ alone is not able to stimulate proton-leak in skeletal 
muscle mitochondria (Lombardi et al. 2008). In agreement, (Bugger et al. 2008) also reported that fatty acid-induced mitochondrial uncoupling in type 1 diabetic hearts required a concomitant increase in the levels of ROS or lipid peroxides. Fasting, high-fat feeding, and STZ-induced diabetes have been demonstrated to increase plasma free fatty acids and a positive correlation between free fatty acids and UCP2 and UCP3 levels in heart (Murray et al. 2005) and liver (Ruiz-Ramirez et al. 2011) mitochondria was observed. It was also previously shown that a severe hypoglycemic episode led to increased levels of free fatty acids in rat cerebral cortex (Agardh and Siesjo 1981). Besides that, previous findings from our laboratory demonstrated that cortical mitochondria from recurrent hypoglycemic animals exhibited higher levels of lipid peroxidation (malondialdehyde) and oxidative stress markers such as decreased mitochondrial aconitase activity (Cardoso et al. 2012). Taking this evidence into account, it is tempting to suggest that the increase in oxidative stress and free fatty acids levels associated to hypoglycemia contribute for the higher sensitivity of mitochondria to UCPs-mediated proton-leak. Our results also suggest that under both metabolic insults, ANT is involved in proton-leak modulation whereas UCPsinduced uncoupling prevails in recurrent hypoglycemia. Still, more studies are needed to clarify the role of neuronal UCPs (UCP2, UCP4, and UCP5) and ANT in hypoglycemia and/or hyperglycemia. Understanding the mechanisms underlying the distinct behavior of mitochondria under different metabolic insults may allow the design of more effective therapeutic strategies.

Acknowledgments The authors' work is supported by the Fundação para a Ciência e a Tecnologia (FCT) (PTDC/SAU-NEU/103325/2008) co-funded by Fundo Europeu de Desenvolvimento Regional (FEDER) via Programa Operacional Factores de Competitividade (COMPETE).

Susana Cardoso has a PhD fellowship from the Portuguese Foundation for Science and Technology (SFRH/BD/43968/2008).

We thank Dra Ana Sofia Rodrigues for her essential assistance with proton-leak measurements and to Dr Gonçalo Pereira and Dra Vilma Sardão for kindling providing the ANT and COX-IV antibodies, respectively.

\section{References}

Agardh CD, Siesjo BK (1981) Hypoglycemic brain injury: phospholipids, free fatty acids, and cyclic nucleotides in the cerebellum of the rat after 30 and $60 \mathrm{~min}$ of severe insulin-induced hypoglycemia. J Cereb Blood Flow Metab 1(3):267-275

Aguirre E, Cadenas S (2010) GDP and carboxyatractylate inhibit 4hydroxynonenal-activated proton conductance to differing degrees in mitochondria from skeletal muscle and heart. Biochim Biophys Acta 1797(10):1716-1726

Boudina S, Sena S, Theobald H, Sheng X, Wright JJ, Hu XX et al (2007) Mitochondrial energetics in the heart in obesity-related diabetes: direct evidence for increased uncoupled respiration and activation of uncoupling proteins. Diabetes 56(10):2457-2466
Brand MD, Esteves TC (2005) Physiological functions of the mitochondrial uncoupling proteins UCP2 and UCP3. Cell Metab 2(2):85-93

Brand MD, Pakay JL, Ocloo A, Kokoszka J, Wallace DC, Brookes PS et al (2005) The basal proton conductance of mitochondria depends on adenine nucleotide translocase content. Biochem $\mathrm{J}$ 392(Pt 2):353-362

Brookes PS (2005) Mitochondrial H(+) leak and ROS generation: an odd couple. Free Radic Biol Med 38(1):12-23

Bugger H, Boudina S, Hu XX, Tuinei J, Zaha VG, Theobald HA et al (2008) Type 1 diabetic akita mouse hearts are insulin sensitive but manifest structurally abnormal mitochondria that remain coupled despite increased uncoupling protein 3. Diabetes 57(11):2924 2932

Cadenas S, Buckingham JA, Samec S, Seydoux J, Din N, Dulloo AG et al (1999) UCP2 and UCP3 rise in starved rat skeletal muscle but mitochondrial proton conductance is unchanged. FEBS Lett 462(3):257-260

Cadenas S, Buckingham JA, St-Pierre J, Dickinson K, Jones RB, Brand MD (2000) AMP decreases the efficiency of skeletalmuscle mitochondria. Biochem J 351(Pt 2):307-311

Cadenas S, Echtay KS, Harper JA, Jekabsons MB, Buckingham JA, Grau E et al (2002) The basal proton conductance of skeletal muscle mitochondria from transgenic mice overexpressing or lacking uncoupling protein-3. J Biol Chem 277(4):2773-2778

Cardoso S, Santos MS, Seica R, Moreira PI (2010) Cortical and hippocampal mitochondria bioenergetics and oxidative status during hyperglycemia and/or insulin-induced hypoglycemia. Biochim Biophys Acta 1802(11):942-951

Cardoso S, Santos RX, Carvalho C, Correia SC, Santos MS, Moreira PI (2011) Mitochondrial uncoupling proteins and oxidative stress: implications for diabetes and neurodegeneration. Free Rad Antiox $1(2): 4-14$

Cardoso S, Santos RX, Correia SC, Carvalho C, Santos MS, Baldeiras I et al (2012) Insulin-induced recurrent hypoglycemia exacerbates diabetic brain mitochondrial dysfunction and oxidative imbalance. Neurobiol Dis 49C:1-12

Chan SL, Wei Z, Chigurupati S, Tu W (2010) Compromised respiratory adaptation and thermoregulation in aging and age-related diseases. Ageing Res Rev 9(1):20-40

Craft S (2009) The role of metabolic disorders in Alzheimer disease and vascular dementia: two roads converged. Arch Neurol 66(3):300-305

Echtay KS, Winkler E, Frischmuth K, Klingenberg M (2001) Uncoupling proteins 2 and 3 are highly active $\mathrm{H}(+)$ transporters and highly nucleotide sensitive when activated by coenzyme Q (ubiquinone). Proc Natl Acad Sci U S A 98(4):1416-1421

Echtay KS, Murphy MP, Smith RA, Talbot DA, Brand MD (2002) Superoxide activates mitochondrial uncoupling protein 2 from the matrix side. Studies using targeted antioxidants. J Biol Chem 277(49):47129-47135

Echtay KS, Esteves TC, Pakay JL, Jekabsons MB, Lambert AJ, Portero-Otin $\mathrm{M}$ et al (2003) A signalling role for 4-hydroxy-2nonenal in regulation of mitochondrial uncoupling. EMBO J 22(16):4103-4110

Gispen WH, Biessels GJ (2000) Cognition and synaptic plasticity in diabetes mellitus. Trends Neurosci 23(11):542-549

Goglia F, Skulachev VP (2003) A function for novel uncoupling proteins: antioxidant defense of mitochondrial matrix by translocating fatty acid peroxides from the inner to the outer membrane leaflet. FASEB J 17(12):1585-1591

Gornall AG, Bardawill CJ, David MM (1949) Determination of serum proteins by means of the biuret reaction. J Biol Chem 177(2):751-766

Herlein JA, Fink BD, O’Malley Y, Sivitz WI (2009) Superoxide and respiratory coupling in mitochondria of insulin-deficient diabetic rats. Endocrinology 150(1):46-55 
Herzog RI, Chan O, Yu S, Dziura J, McNay EC, Sherwin RS (2008) Effect of acute and recurrent hypoglycemia on changes in brain glycogen concentration. Endocrinology 149(4):1499-1504

Hoerter J, Gonzalez-Barroso MD, Couplan E, Mateo P, Gelly C, Cassard-Doulcier AM et al (2004) Mitochondrial uncoupling protein 1 expressed in the heart of transgenic mice protects against ischemic-reperfusion damage. Circulation 110(5):528-533

Jaburek M, Miyamoto S, Di Mascio P, Garlid KD, Jezek P (2004) Hydroperoxy fatty acid cycling mediated by mitochondrial uncoupling protein UCP2. J Biol Chem 279(51):53097-53102

Jacob RJ, Dziura J, Blumberg M, Morgen JP, Sherwin RS (1999) Effects of recurrent hypoglycemia on brainstem function in diabetic BB rats: protective adaptation during acute hypoglycemia. Diabetes 48(1):141-145

Korshunov SS, Skulachev VP, Starkov AA (1997) High protonic potential actuates a mechanism of production of reactive oxygen species in mitochondria. FEBS Lett 416(1):15-18

Leloup C, Casteilla L, Carriere A, Galinier A, Benani A, Carneiro L et al (2011) Balancing mitochondrial redox signaling: a key point in metabolic regulation. Antioxid Redox Signal 14(3):519-530

Lombardi A, Grasso P, Moreno M, de Lange P, Silvestri E, Lanni A et al (2008) Interrelated influence of superoxides and free fatty acids over mitochondrial uncoupling in skeletal muscle. Biochim Biophys Acta 1777(7-8):826-833

Mattiasson G, Shamloo M, Gido G, Mathi K, Tomasevic G, Yi S et al (2003) Uncoupling protein-2 prevents neuronal death and diminishes brain dysfunction after stroke and brain trauma. Nat Med 9(8):10621068

McGowan JE, Chen L, Gao D, Trush M, Wei C (2006) Increased mitochondrial reactive oxygen species production in newborn brain during hypoglycemia. Neurosci Lett 399(1-2):111-114

Miwa S, Brand MD (2003) Mitochondrial matrix reactive oxygen species production is very sensitive to mild uncoupling. Biochem Soc Trans 31(Pt 6):1300-1301

Moreira PI, Santos MS, Moreno A, Oliveira C (2001) Amyloid betapeptide promotes permeability transition pore in brain mitochondria. Biosci Rep 21(6):789-800

Moreira PI, Santos MS, Moreno A, Rego AC, Oliveira C (2002) Effect of amyloid beta-peptide on permeability transition pore: a comparative study. J Neurosci Res 69(2):257-267

Moreira PI, Santos MS, Sena C, Seica R, Oliveira CR (2005) Insulin protects against amyloid beta-peptide toxicity in brain mitochondria of diabetic rats. Neurobiol Dis 18(3):628-637

Moreira PI, Cardoso SM, Pereira CM, Santos MS, Oliveira CR (2009) Mitochondria as a therapeutic target in Alzheimer's disease and diabetes. CNS Neurol Disord Drug Targets 8(6):492-511

Murphy MP, Echtay KS, Blaikie FH, Asin-Cayuela J, Cocheme HM, Green K et al (2003) Superoxide activates uncoupling proteins by generating carbon-centered radicals and initiating lipid peroxidation: studies using a mitochondria-targeted spin trap derived from alpha-phenyl-N-tert-butylnitrone. J Biol Chem 278(49):48534 48545

Murray AJ, Panagia M, Hauton D, Gibbons GF, Clarke K (2005) Plasma free fatty acids and peroxisome proliferator-activated receptor alpha in the control of myocardial uncoupling protein levels. Diabetes 54(12):3496-3502

Naudi A, Jove M, Ayala V, Cassanye A, Serrano J, Gonzalo H et al (2012) Cellular dysfunction in diabetes as maladaptive response to mitochondrial oxidative stress. Exp Diabetes Res 2012:696215

Negre-Salvayre A, Hirtz C, Carrera G, Cazenave R, Troly M, Salvayre R et al (1997) A role for uncoupling protein-2 as a regulator of mitochondrial hydrogen peroxide generation. FASEB J 11(10): 809-815

Nishikawa T, Edelstein D, Du XL, Yamagishi S, Matsumura T, Kaneda $Y$ et al (2000) Normalizing mitochondrial superoxide production blocks three pathways of hyperglycaemic damage. Nature 404(6779):787-790

Rabol R, Hojberg PM, Almdal T, Boushel R, Haugaard SB, Madsbad S et al (2009) Improved glycaemic control decreases inner mitochondrial membrane leak in type 2 diabetes. Diabetes Obes Metab 11(4):355-360

Rains JL, Jain SK (2011) Oxidative stress, insulin signaling, and diabetes. Free Radic Biol Med 50(5):567-5

Ruiz-Ramirez A, Chavez-Salgado M, Peneda-Flores JA, Zapata E, Masso F, El-Hafidi M (2011) High-sucrose diet increases ROS generation, FFA accumulation, UCP2 level, and proton leak in liver mitochondria. Am J Physiol Endocrinol Metab 301(6): E1198-E1207

Stuart JA, Brindle KM, Harper JA, Brand MD (1999) Mitochondrial proton leak and the uncoupling proteins. J Bioenerg Biomembr 31(5):517-525

Teshima Y, Akao M, Jones SP, Marban E (2003) Uncoupling protein-2 overexpression inhibits mitochondrial death pathway in cardiomyocytes. Circ Res 93(3):192-200

Trzcionka M, Withers KW, Klingenspor M, Jastroch M (2008) The effects of fasting and cold exposure on metabolic rate and mitochondrial proton leak in liver and skeletal muscle of an amphibian, the cane toad Bufo marinus. J Exp Biol 211(Pt 12):19111918

Vincent AM, Olzmann JA, Brownlee M, Sivitz WI, Russell JW (2004) Uncoupling proteins prevent glucose-induced neuronal oxidative stress and programmed cell death. Diabetes 53(3):726-734

Votyakova TV, Reynolds IJ (2001) DeltaPsi(m)-Dependent and independent production of reactive oxygen species by rat brain mitochondria. J Neurochem 79(2):266-277

Wrighten SA, Piroli GG, Grillo CA, Reagan LP (2009) A look inside the diabetic brain: contributors to diabetes-induced brain aging. Biochim Biophys Acta 1792(5):444-453 